\title{
Short Communication: Difficulties in Estimating Across-Country Genetic Correlations for Weakly Linked Bull Populations
}

\author{
T. Mark, ${ }^{1}$ P. Madsen, ${ }^{2}$ J. Jensen, ${ }^{2}$ and W. F. Fikse ${ }^{1}$ \\ ${ }^{1}$ Interbull Centre, Department of Animal Breeding and Genetics, Swedish University of Agricultural Sciences, \\ 75007 Uppsala, Sweden \\ ${ }^{2}$ Danish Institute of Agricultural Sciences, Research Centre Foulum, 8830 Tjele, Denmark
}

\begin{abstract}
Genetic correlations across 9 weakly linked bull populations were estimated with various models, methods, and data sets. Estimates of the same genetic correlation differed noticeably depending on estimation strategy (up to 1.03 units) for country-pairs with no or few direct genetic links. Differences in estimated genetic correlations decreased with an increasing number of genetic ties between countries, and, essentially, no differences were found between estimated genetic correlations based on 61 common bulls, regardless of the estimation strategy used.

(Key words: genetic correlation, estimation, multipletrait across-country evaluation, poor connectedness)
\end{abstract}

Abbreviation key: AI-REML = average-informationREML, $\mathbf{C B}=$ number of bulls with evaluations in multiple countries, EM-REML = expectation-maximization REML, MACE = multiple-trait, across-country evaluations, $\mathbf{r}_{\mathbf{G}}=$ across-country genetic correlation.

Estimation of across-country genetic correlations $\left(\mathbf{r}_{\mathbf{G}}\right)$ is difficult, mainly because the majority of bulls have daughters in only one country. Estimation is further complicated by less information being available to infer residual (co)variances when deregressed national breeding values are used as dependent variables instead of individual performance data. Therefore, heritabilities in Interbull's international genetic evaluations are usually assumed to be equal to the heritabilities used in the national genetic evaluations.

Mark et al. (2005) obtained $r_{G}$ with both expectationmaximization REML (EM-REML) and Gibbs sampling for the purpose of comparing traditional multiple-trait across-country evaluations (MACE; Schaeffer, 1994) and a fully Bayesian MACE implemented via Gibbs sampling. Those researchers observed noticeable differ-

Received February 4, 2005.

Accepted April 26, 2005.

Corresponding author: T. Mark; e-mail: Thomas.Mark@hgen. slu.se. ences in estimated $\mathrm{r}_{\mathrm{G}}$. However, from their study, it was not clear to what extent the differences in estimated $r_{G}$ were caused by differences in the data considered, the models used, and the assumed priors. Thus, the aim of this study was to evaulate the impact of different model assumptions and choice of data on estimated $r_{G}$ between weakly linked bull populations.

The data used for this purpose were identical to the data described by Mark et al. (2005) (i.e., data consisted of deregressed national genetic bull evaluations for fore udder attachment from 9 weakly linked Ayrshire populations). Two data sets were considered (i.e., the full data set consisting of 5913 records and a well-connected subset consisting of 455 records). The well-connected subset consisted of bulls with evaluations in more than one country (common bulls; CB) as well as bulls belonging to $3 / 4$ sib groups that have members with evaluations in more than one country. The number of bulls with evaluations in $2,3,4,5$, and 6 countries was 102 , $21,5,3$, and 1 , respectively.

Genetic correlations were estimated in 5 different MACE analyses (Table 1). The first analysis (run A) was by the EM-REML algorithm of Klei and Weigel (1998), which was applied to the well-connected subset. This analysis assumed random genetic group effects and national heritabilities; it corresponded to current Interbull practice, and the estimated $\mathrm{r}_{\mathrm{G}}$ were identical to those used by Mark et al. (2005). Run B was similar to run A, except that it was based on the full data set. Run C was similar to run B, except that genetic groups were treated as a fixed effect instead of a random effect. Run D was similar to run C, except that heritabilities were estimated instead of being kept fixed at the national assumptions during estimation. Run D was implemented in the DMU software package, and the REML log-likelihood was optimized using average-information REML (AI-REML) (Madsen et al., 2000); however, switching to an EM algorithm to avoid updates falling outside their allowed parameter space. Run E was Gibbs sampling using Holstein genetic correlations as prior means. This analysis was identical to Run 10 as described by Mark et al. (2005) (i.e., 5 df was assumed for the inverse-Wishart distribution for prior 
Table 1. Summary of analyses and average across-country genetic correlations $\left(\mathrm{r}_{\mathrm{G}}\right)$.

\begin{tabular}{llllll}
\hline Run & Method & $\mathrm{h}^{2}$ & Group $^{1}$ & Data & $\mathrm{r}_{\mathrm{G}}$ \\
\hline A & REML EM $^{2}$ & Fixed & Random & Subset $^{3}$ & 0.56 \\
B & REML EM $^{2}$ & Fixed & Random & All $^{4}$ & 0.57 \\
C & REML EM $^{2}$ & Fixed & Fixed & All & 0.64 \\
D & REML AI/EM & Estimated & Fixed & All & 0.71 \\
E & Gibbs $^{6}$ & Estimated & Fixed & All & 0.79 \\
\hline
\end{tabular}

${ }^{1}$ Assumption regarding genetic group effects.

${ }^{2} \mathrm{EM}=$ Expectation-maximization (Klei and Weigel, 1998).

${ }^{3}$ Well-connected subset of bulls.

${ }^{4}$ All bulls with daughters in $\geq 10$ herds.

${ }^{5} \mathrm{AI}=$ Average information (Madsen et al., 2000).

${ }^{6}$ Mean posterior $\mathrm{r}_{\mathrm{G}}$ obtained by Gibbs sampling [Holstein $\mathrm{r}_{\mathrm{G}}$ used as priors; average prior mean was 0.72 and $5 \mathrm{df}$ as prior belief; identical to run 10 in Mark et al. (2005)].

(co)variances). Asymptotic standard errors $\sigma_{\mathrm{rG}}$ of estimated $\mathrm{r}_{\mathrm{G}}$ (Madsen et al., 2000) were calculated for run $\mathrm{D}$ and were used to interpret the magnitude of differences in estimated $r_{G}$ caused by different model assumptions and data used.

Using all data in comparison with a well-connected subset had a noticeable impact on some estimated $r_{G}$ for country-pairs with few or no direct genetic links (Figure 1A). The largest difference between estimates was 0.9 , but, on average, the estimated $r_{G}$ were only 0.01 units higher when based on all data compared with when a well-connected subset was used for correlation estimation. The differences may be explained by selection bias when a well-connected subset is used.

Treatment of genetic groups as either fixed or random effects in MACE had some impact on estimated $r_{G}$ even
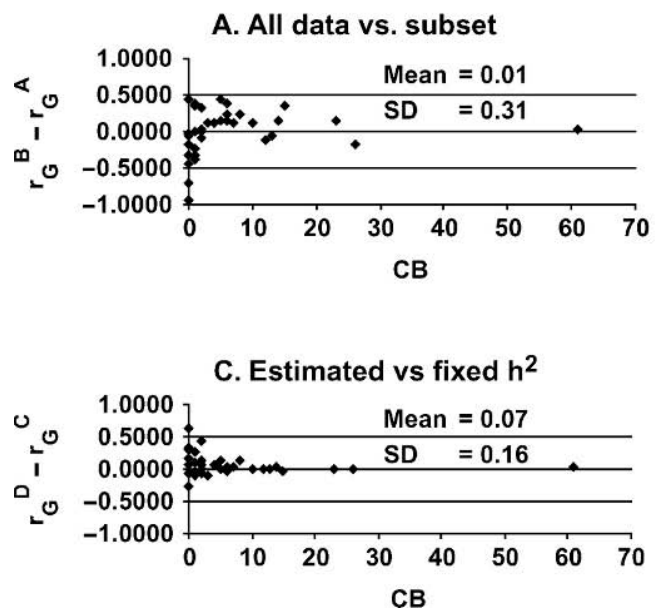

though group sizes were relatively large (i.e. $>500$ phantom parents per group). The reason for this could be that although group sizes were large, the majority of phantom parents mainly provided information about the group effect in one country (e.g., there was only little direct and indirect information available to estimate the genetic group effect for maternal grandams of Norwegian origin on non-Norwegian country scales). Having genetic groups as fixed effects resulted in much slower convergence, and $\mathrm{r}_{\mathrm{G}}$ converged to different local optima depending on the starting values used. Runs B and D did not suffer from these convergence problems. The assumption about genetic group effects clearly had a larger impact on estimated $r_{G}$ for country-pairs with few or no direct genetic links compared with countrypairs with $>10 \mathrm{CB}$. The differences in Figure 1B may also be due to the EM-REML algorithm of Klei and Weigel (1998) not optimizing the complete likelihood when genetic group effects are assumed random.

The impact of assuming either fixed or estimated heritabilities in MACE was relatively small for most estimated genetic correlations. The impact was smaller compared with both the impact of which data that were used and the impact of different treatment of genetic group effects. However, estimated $r_{G}$ obtained while assuming estimated heritabilities were on average 0.07 larger than estimated $\mathrm{r}_{\mathrm{G}}$ obtained for a model assuming heritabilities equal to those used in national genetic evaluations. The main difference between estimated $\mathrm{r}_{\mathrm{G}}$ (Figure 1C) should be the assumptions regarding heritability. However, the differences could also be due to the 2 different models being implemented in different
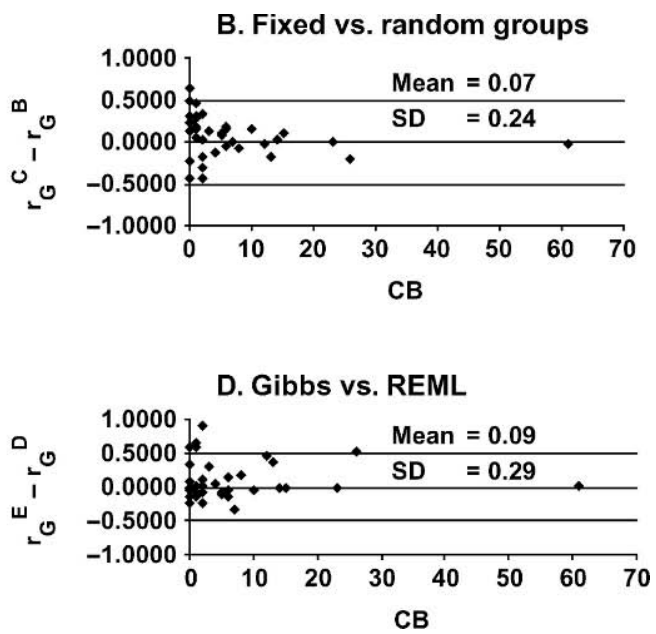

Figure 1. Differences $($ ) between genetic correlations inferred with different methods or based on different data sets as a function of number of common Ayrshire bulls $(\mathrm{CB}) \cdot \mathrm{r}_{\mathrm{G}}{ }^{\mathrm{A}}=$ genetic correlations estimated with REML for a model assuming fixed $\mathrm{h}^{2}$ and random genetic groups applied to a well-connected subset of data; $r_{G}{ }^{B}=r_{G}{ }^{A}$ except estimation is based on all data; $r_{G}{ }^{C}=r_{G}{ }^{B}$ except model assumes fixed genetic groups; $r_{G}{ }^{D}=r_{G}{ }^{C}$ except heritabilities were estimated; $r_{G}{ }^{E}=$ posterior means of genetic correlations inferred with Gibbs sampling. 
software packages. The optimization with AI-REML switched to an EM algorithm after 11 rounds of iteration, and EM updates were obtained until convergence. Estimated $\mathrm{r}_{\mathrm{G}}$ for country-pairs with $>10 \mathrm{CB}$ were essentially the same regardless of whether heritabilities were kept fixed or not during the estimation.

Posterior means of $r_{G}$ from Gibbs sampling were on average 0.09 units higher than $r_{G}$ estimated with AIREML; prior means were only 0.01 units higher than the AI-REML estimates on average. The main reason for this was that the difference between prior means and REML estimates tended to be larger for countrypairs with zero or low CB compared with country-pairs with higher CB. Thus, the posterior means of $r_{G}$ closely followed the prior means (i.e., the largest difference was 0.2 units, and the standard deviation of differences was 0.07 units for posterior vs. prior means). This explains why increasing the prior belief from 5 to 100 degrees of freedom had a relatively small impact on posterior means of $r_{G}$ (i.e., the largest difference was 0.1 units, and the standard deviation of differences was 0.05) (results not shown).

The largest absolute difference in estimated $r_{G}$ (Figure 1) relative to asymptotic standard error of estimated $\mathrm{r}_{\mathrm{G}}$ was 7.9 units. However, 94, 88, and $78 \%$ of all absolute differences in estimated $r_{G}$ were lower than 2,1 , and 0.5 units of $\sigma_{\mathrm{rG}}$, respectively.

The weighting factors used in MACE (effective daughter contributions; Fikse and Banos, 2001) are scaled according to the heritabilities assumed in MACE. In principle, these weighting factors should be re-scaled according to the re-estimated heritatibilites during iteration. However, Fikse and Banos (2001) found that estimated $\mathrm{r}_{\mathrm{G}}$ were unaffected by the choice of weighting factor, so the re-scaling is probably not necessary for the purpose of estimating $\mathrm{r}_{\mathrm{G}}$.

The average prior mean of $r_{G}$ was 0.72 (i.e., higher than the average REML estimates, but lower than the average posterior mean $\mathrm{r}_{\mathrm{G}}$ obtained by Gibbs sampling). The large variation in estimated $r_{G}$ for different estimation strategies was not expected although $\mathrm{r}_{\mathrm{G}}$ for country-pairs with few direct genetic links tended to differ most.

The large variation in estimated $r_{G}$ caused by estimation strategy illustrates the difficulty of estimating $r_{G}$ when the number of bulls with multiple evaluations is low. The actual level of differences may vary depending on the true $\mathrm{r}_{\mathrm{G}}$, heritabilities, as well as population characteristics other than $\mathrm{CB}$, as the precision of the estimated $r_{G}$ depends on these factors (Robertson, 1959). However, similar tendencies for the differences in estimated $r_{G}$ as a function of CB are also expected for other traits and populations. Generally, the differences tended to decrease with an increasing amount of genetic ties between countries. The estimated $r_{G}$ were not affected much by the differences in method, model, and data used when CB was sufficiently large $(>20)$. This was best illustrated for the $r_{G}$ between Canada and the US, which was based on the highest $\mathrm{CB}$ (i.e., $\mathrm{CB}=61$ ).

\section{REFERENCES}

Fikse, W. F., and G. Banos. 2001. Weighting factors of sire daughter information in international genetic evaluations. J. Dairy Sci. 84:1759-1767.

Klei, B., and K. A. Weigel. 1998. A method to estimate correlations among traits in different countries using data on all bulls. Proc. 1998 Interbull Mtg., Rotorua, New Zealand. Interbull Bull. 17:8-14.

Madsen, P., J. Jensen, and T. Mark. 2000. Reduced rank estimation of (co)variance components for international evaluation using AIREML. Proc. 2000 Interbull Mtg., Bled, Slovenia. Interbull Bull. 25:46-50.

Mark, T., P. Madsen, J. Jensen, and W. F. Fikse. 2005. Prior (co)variances can improve multiple-trait across country evaluations of weakly linked bull populations J. Dairy Sci. 88:3290-3302.

Robertson, A. 1959. The sampling variance of the genetic correlation coefficient. Biometrics 15:469-485.

Schaeffer, L. R. 1994. Multiple-country comparisons of dairy sires. J. Dairy Sci. 77:2671-2678. 